\begin{tabular}{|c|c|}
\hline Title & $\begin{array}{l}\text { Reliability of an ultrasonographical scoring sy stem for diagnosis of sinusoidal obstruction syndromeNeno-occlusive } \\
\text { disease in patients with hematopoietic stem cell transplantation }\end{array}$ \\
\hline Author(s) & $\begin{array}{l}\text { Iwai, Takahito; Nishida, Mutsumi; Sugita, Junichi; Kudo, Y usuke; Takasugi, Rika; Y okota, I sao; Takagi, Ryo; Shibuya, } \\
\text { Hitoshi; T Takahashi, Shuichiro; Teshima, Takanori }\end{array}$ \\
\hline Citation & $\begin{array}{l}\text { Journal of medical ultrasonics, } 48(1), 45-52 \\
\text { https://doi.org/10.1007/s10396-020-01071-1 }\end{array}$ \\
\hline Issue Date & 2021-01 \\
\hline Doc URL & http:/hdl. handle.net/2115/83793 \\
\hline Rights & $\begin{array}{l}\text { This is a post-peer-review, pre copy edit version of an article published in Journal of medical ultrasonics. The final } \\
\text { authenticated version is avail able online at: http://dx.doi.org/10.1007/s10396-020-01071-1 }\end{array}$ \\
\hline Type & article (author version) \\
\hline File Information & J Med UItrason 48_45.pdf \\
\hline
\end{tabular}

Instructions for use 


\section{Reliability of an ultrasonographical scoring system for diagnosis of sinusoidal obstruction syndrome / veno-occlusive disease in patients with hematopoietic stem cell transplantation}

Takahito Iwai ${ }^{1,2}$; Mutsumi Nishida, $\mathrm{PhD}^{1,2}$; Junichi Sugita, $\mathrm{MD}, \mathrm{PhD}^{1,3}$; Yusuke Kudo, $\mathrm{PhD}^{1,2}$; Rika Takasugi ${ }^{2}$; Isao Yokota, $\mathrm{PhD}, \mathrm{MPH}^{4}$; Ryo Takagi ${ }^{5}$; Hitoshi Shibuya ${ }^{2}$; Shuichiro Takahashi, $\mathrm{MD}, \mathrm{PhD}^{1,3}$; and Takanori Teshima, $\mathrm{MD}, \mathrm{PhD}^{1,3}$

${ }^{1}$ Division of Laboratory and Transfusion Medicine, Hokkaido University Hospital, Sapporo, Japan

${ }^{2}$ Diagnostic Center for Sonography, Hokkaido University Hospital, Sapporo, Japan

${ }^{3}$ Department of Hematology, Hokkaido University Hospital, Sapporo, Japan

${ }^{4}$ Department of Biostatistics, Graduate School of Medicine, Hokkaido University, Sapporo, Japan

${ }^{5}$ Biostatistics Division, Hokkaido University Hospital Clinical Research and Medical Innovation Center, Sapporo, Japan

Corresponding author: Mutsumi Nishida, Ph.D.

Division of Laboratory and Transfusion Medicine, Hokkaido University Hospital N14 W5, Kita-ku, Sapporo 060-8648, Japan

Tel: +81-11-706-5697

Fax: +81-11-706-7614

E-mail: mutuni@med.hokudai.ac.jp 


\section{Abstract}

2 Purpose: Sinusoidal obstruction syndrome (SOS) / hepatic veno-occlusive disease (VOD)

3 is a fatal complication after hematopoietic stem cell transplantation. We previously

4 reported the usefulness of an ultrasonographical (US) scoring system, the Hokkaido

5 US-based scoring system consisting of 10 parameters (HokUS-10): 1) hepatomegaly in

6 the left lobe and 2) right lobe, 3) dilatation of the main portal vein (PV), 4) hepatofugal

7 flow in the main PV, 5) decreased velocity of the PV, 6) dilatation of the para-umbilical

8 vein (PUV), 7) appearance of blood flow signal in the PUV, 8) gallbladder (GB) wall

9 thickening, 9) ascites, and 10) increased resistive index of the hepatic artery, for the

10 diagnosis of SOS/VOD. However, the reliability of this system among operators remains

11 elusive. Therefore, we prospectively evaluated the reliability of HokUS-10.

12 Methods: Twenty-four healthy volunteers and 40 patients with liver dysfunction were

13 enrolled. Inter- and intra-operator reliabilities were analyzed using three sonographers.

14 Results: The median concordance rate of HokUS-10 among three sonographers and

15 intra-operator in 24 volunteers was 92\% (95\% CI: 73-98\%) and 98\% (95\% CI: 92-100\%),

16 respectively. In all 64 cases, in terms of the reliability between two sonographers for

17 three representative US parameters (amount of ascites, GB wall thickening, and

18 appearance of PUV blood flow signal), the median concordance rate was more than 98\%

19 (95\% CI: 86-106\%).

20 Conclusion: The inter- and intra-reliabilities of HokUS-10 were excellent. Thus, US

21 might be a reliable tool for SOS/VOD diagnosis.

\section{Keywords}

24 sinusoidal obstruction syndrome, hepatic veno-occlusive disease, hematopoietic stem cell

25 transplantation, operator reliability, ultrasonography 


\section{Introduction}

2 Sinusoidal obstruction syndrome (SOS) / hepatic veno-occlusive disease (VOD) is a fatal

3 complication mainly arising after hematopoietic stem cell transplantation (HSCT) [1].

4 Chemotherapy and/or radiotherapy for cancer treatment or a conditioning regimen of

5 HSCT can cause hepatic sinusoidal cell damage leading to sinusoidal obstruction, which

6 increases the downstream pressure and may lead to portal hypertension. Severe

7 SOS/VOD has a high mortality rate $>80 \%$ due to multiple organ failure [2].

8 Clinically, the Baltimore and modified Seattle criteria have been used for the diagnosis

9 of SOS/VOD, which consist of high bilirubin level ( $\geq 2 \mathrm{mg} / \mathrm{dl}$ ), ascites, right upper

10 quadrant pain, and increasing body weight appearing within 21 days after HSCT.

11 However, SOS/VOD is often difficult to diagnose because weight gain and

12 hyperbilirubinemia can occur for reasons other than SOS/VOD.

13 Ultrasonography (US) was reported as a useful tool to diagnose SOS/VOD, but only in 14 two prospective studies [3, 4] and some case reports, most of which were published more 15 than 20 years ago [3-11]. Recently, US has been incorporated in the European Blood and 16 Marrow Transplant (EBMT) diagnostic criteria of SOS/VOD [12, 13]. Concurrently, we 17 previously reported the usefulness of the Hokkaido US-based scoring system 18 (HokUS-10) consisting of 10 parameters for SOS/VOD diagnosis in a prospective 19 manner [14] (Table 1). Since then, HokUS-10 has been cited in several articles [15-19]; 20 however, it has not been validated [19].

21 In this study, we prospectively analyzed the operator reliability as an intra-facility 22 validation for HokUS-10. 


\section{Materials and Methods}

\section{Study population}

In this study, healthy volunteers with no liver dysfunction were enrolled between March 2017 and March 2019 based on their most recent medical examination. Patients diagnosed with liver dysfunction at the Hokkaido University Hospital were also enrolled. Fasting over $4 \mathrm{~h}$ was required for healthy volunteers and patients.

\section{Transabdominal ultrasonography}

US was performed using a PVT-375 BT (center frequency, $3.75 \mathrm{MHz}$ ), PVT-674 BT (center frequency, $6 \mathrm{MHz}$ ), and PVT-704 BT (center frequency, 7.5 MHz) attached to the Aplio $^{\mathrm{TM}}$ XG (Canon Medical Systems Corp., Otawara, Japan).

\section{Healthy volunteers}

The inclusion criteria for healthy volunteers were as follows: age above 20 years, no history of liver dysfunction, and ability to undergo two US examinations within 1 week. Ten HokUS-10 parameters, which consist of B-mode and Doppler findings, were evaluated: hepatomegaly in the (1) left lobe ( $\geq 70 \mathrm{~mm}$ ) and (2) right lobe ( $\geq 110 \mathrm{~mm})$; (3) gallbladder (GB) wall thickening ( $\geq 6 \mathrm{~mm}$ ) [Fig. 1]; (4) dilatation of the main portal vein $(\mathrm{PV} \geq 12 \mathrm{~mm}$ ); (5) dilatation of the paraumbilical vein ( $\mathrm{PUV} \geq 2 \mathrm{~mm}$ ); (6) moderate amount of ascites defined by the presence of ascites in the Douglas pouch, subhepatic space, and spleno-renal interspace (maximum thickness $\geq 10 \mathrm{~mm}$ in least two sites) [Fig. 2]; (7) decreased mean velocity of the PV (time-averaged flow velocity [TAV], $<10$ $\mathrm{cm} / \mathrm{s}$ ); (8) congestion or hepatofugal flow in the main PV; (9) appearance of a blood flow signal in the PUV (congestion or hepatofugal flow) [Fig. 3]; and (10) increased resistive index (RI) of the proper hepatic artery (PHA, $\geq 0.75$ ).

The anteroposterior diameter of (1) the left liver lobe was measured in front of the abdominal aorta so that the caudate lobe was not usually included. The anteroposterior diameter of (2) the right liver lobe was measured in front of the right kidney and on the anterior clavicular line. (3) GB wall thickening was measured using the intercostal or subcostal approach in the thickest part, avoiding lesions such as adenomyomatosis and polyps. The diameters of (4) the main PV and (5) PUV were measured using the subcostal approach. The diameters of (1) the left liver lobe, (2) right lobe, (3) GB wall thickening, and (4) main PV were measured from one leading edge to another, while the diameter of (5) the PUV was measured with the endo-cavity interface.

The velocity of (7) the PV was determined with an auto-trace measurement delineated with the subcostal approach, and an insonation angle was set at less than 60 degrees, with the sample gate set at more than 2/3 of the vessel diameter. (10) Measurement of the RI in 
1 the PHA should be able to accurately recognize the maximum flow velocity $\left(\mathrm{V}_{\max }\right)$ and

2 the minimum flow velocity $\left(\mathrm{V}_{\mathrm{min}}\right)$. Pulse-wave Doppler measurement was performed

3 with shallow breath holding, and sensitivities and thresholds were properly adjusted.

HokUS-10 was evaluated on the same day by three sonographers ([A] M.N. with 33 years of experience, [B] Y.K. with 7 years of experience, and [C] R.T. with 2 years of experience) in random order. All sonographers were blinded to each other's results.

7 Repetitive US for HokUS-10 was conducted by sonographer B within 1 week.

8 Additionally, the duration of each examination was recorded and compared among the

9 three sonographers.

\section{Patients}

After undergoing hematopoietic stem cell transplantation, patients are mostly in a sterile room and their condition is not good. Also, US findings of SOS/VOD are similar to liver cirrhosis and portal hypertension. Thus, we enrolled patients older than 20 years with liver dysfunction instead of SOS/VOD patients. We excluded patients who were unable to undergo a second US examination, required oxygen, and/or could not move independently.

Performing US three times and measuring the same 10 items each time in the same patient is not practical as it takes a considerable amount of time and is a burden to the patient. To minimize the burden placed on the patients, we selected three critical parameters to diagnose SOS/VOD. (1) GB wall thickening, (2) amount of ascites, and (3) appearance of a blood flow signal in the PUV of HokUS-10 were evaluated in patients with liver dysfunction on the same day by two sonographers in random order (A and B). The two sonographers were blinded to each other's results.

\section{Re-assessments}

Another sonographer ([D] T.I. with 5 years of experience) subsequently remeasured or reassessed all still images of the healthy volunteers blindly obtained by the first three sonographers. Five parameters ((1) the left and (2) right lobes of the liver, (3) GB wall thickening, (4) the main PV diameter, and (5) the PUV diameter) were remeasured on the monitor of the US machine. The RI of the PHA was calculated using proportional calculation after measuring the lengths of $\mathrm{V}_{\max }$ and $\mathrm{V}_{\min }$ by placing a ruler on the monitor of an offline personal computer. The mean velocity of the PV (TAV) was calculated by multiplying the time-averaged maximum velocity (TAMV) by a coefficient of 0.57 [20] on still images since the TAV of PV can only be measured in real time. In addition, the amount of ascites, congestion or hepatofugal flow in the main PV, and appearance of a blood flow signal in the PUV were reassessed using stored still US images. 
1

\section{Statistical analysis}

3 Each HokUS-10 parameter was evaluated by the concordance rate and/or the kappa

4

5

6 coefficient. The concordance rate is the value where the score of each item matches in the inter- and intra-operator assessment. HokUS-10's inter-operator reliability was evaluated with the concordance rate and Krippendorff's alpha coefficient (ordinal), and its intra-operator reliability was evaluated with the concordance rate and weighted kappa coefficient. The kappa coefficient and Krippendorff's alpha values were interpreted as follows: 0.00-0.20, slight agreement; 0.21-0.40, fair agreement; 0.41-0.60, moderate agreement; 0.61-0.80, substantial agreement; and 0.81-1.00, almost perfect agreement [21]. All statistical analyses were performed using standard statistical software (SPSS Statistics Version 23.0 [IBM, Armonk, NY, USA], BellCurve for Excel Version 2.11 [Social Survey Research Information Co., Ltd., Tokyo, Japan], and R [version 3.5.2. http://www.Rproject.org/]).

\section{Results}

One volunteer was excluded because she could not undergo a second US examination within 1 week. Thus, 24 healthy volunteers and 40 patients were enrolled. The characteristics of the study population are shown in Table 2.

\section{Inter-operator reliability of HokUS-10}

The inter-operator reliability in healthy volunteers is shown in Table 3. The concordance rate was higher than $80 \%$ for hepatic diameter in the left lobe, diameter of the main PV, blood flow direction in the main PV, dilatation of the PUV, appearance of a blood flow signal in the PUV, GB wall thickening, and existence/amount of ascites. The concordance rate for hepatic diameter in the right lobe, PV mean velocity, and RI of the PHA were 71\%, 63\%, and 58\%, respectively. Agreement could be evaluated by Fleiss’ kappa coefficient for five items. RI of the PHA showed low agreement (0.16); however, the other four US items showed moderate to almost perfect agreement. The concordance rate (with a cutoff value of 5 or more) and Krippendorff's alpha coefficient of HokUS-10 were $100 \%$ and 0.63 , respectively.

The examination times of sonographers A and B were significantly faster than that of sonographer $\mathrm{C}$. The median examination times of sonographers $\mathrm{A}, \mathrm{B}$, and $\mathrm{C}$ were $9.8 \mathrm{~min}$ (range, 5.5-15.9 min), $8.2 \mathrm{~min}$ (range, 5.8-15.4 $\mathrm{min}$ ), and $12.6 \mathrm{~min}$ (range, 5.9-23.2 $\mathrm{min}$ ), respectively.

\section{Intra-operator reliability of HokUS-10}


1 The intra-operator reliability in healthy volunteers is shown in Table 4. The intra-operator

2 reliability was very good, with a higher than $88 \%$ concordance rate for all 10 items, and

3 there were no items with a significantly lower kappa coefficient. The HokUS-10

4 concordance rate and weighted kappa coefficient showed almost perfect agreement,

$5 \quad 100 \%$ and 0.87 , respectively.

6 The examination times did not differ between the first and second US examinations.

7 The median examination times of the first and second examinations were $8.2 \mathrm{~min}$ (range,

$8 \quad$ 5.8-15.4 $\mathrm{min}$ ) and 9.4 $\mathrm{min}$ (range, 5.3-14.0 $\mathrm{min}$ ), respectively.

\section{Inter-operator reliability of three US criteria}

The inter-operator reliability in all study participants is shown in Table 5. The inter-operator reliability was very good, with a higher than $91 \%$ concordance rate. There was high agreement for all three US criteria. The kappa coefficient of the amount of ascites and appearance of a PUV blood flow signal showed almost perfect agreement, while GB wall thickening showed substantial agreement.

\section{Re-assessments}

18 Remeasurements in healthy volunteers are shown in Table 6. No parameters showed significant differences in the measured values between all three sonographers and sonographer D. There was no significant difference between these measurements. The concordance rate (with a cutoff value of 5 or more) and Krippendorff's alpha coefficient of HokUS-10 were $100 \%$ and 0.64 , respectively.

\section{Discussion}

The inter-operator reliability among three sonographers with varying years of experience was primarily good. A low tendency of differences in the measurements of the right lobe, PV velocity, and RI of the PHA could have resulted from differences in selecting the measurement position, setting the Doppler angle, and the degree of breath-holding in the subjects. In the measurement of RI of the PHA, the differences could have occurred due to the adjustment of the threshold degree in auto-tracing. In some cases, due to the deeper location of the PHA and interference of intestinal gas, the Doppler wave form became obscure, and auto-tracing could not accurately detect the peak and end-diastolic velocity.

To improve accuracy, it is recommended to measure RI using the intercostal approach instead of the subcostal one. Measurement of PV mean velocity is highly influenced by several factors. First, Doppler US for measuring velocity requires the insonation angle to be within 60 degrees; otherwise, overestimation can occur. For instance, the overestimation error becomes $50 \%$ at 80 degrees, and 15\% at 60 degrees [24]. The PV 
1 main trunk naturally runs vertical to the Doppler insonation angle. Operators try to set the 2 angle at less than 60 degrees by tilting the probe and/or applying pressure. The degree of

3 tilting and pressure may sometimes slightly differ among operators, which may result in

4 different velocity values. Second, the PV main trunk is located at a deeper area and

5 intestinal gas can easily interfere, as is the case for the PHA. The above may interfere

6 with accurate measurements but could be expected to improve with use of the intercostal 7 approach [22, 23].

8 Dilatation and the appearance of PUV blood flow signal are important findings, and

9 they showed good agreement. Consequently, the intra-operator reliability was excellent 10 (85\%) or had a higher agreement rate for all items.

11 There was no difference among the three sonographers' measurements and

12 re-measurements by sonographer $\mathrm{D}$; most of the re-measurements and assessments

13 matched well. This result suggests that measurements and assessment of US images can

14 be performed afterward, when the US images are correctly captured. Overall, the scoring

15 of the HokUS-10 was generally consistent between both inter- and intra-operators.

16 There were a few limitations. First, this study was mainly conducted with healthy 17 volunteers. Since a few items showed positive scores, we could not perform statistical 18 analyses for other items, which were ascites, direction of PV flow, and appearance of 19 PUV blood flow signals. Second, this study was conducted at a single institution. In the 20 future, larger multicenter population-based studies are warranted to confirm the validity 21 of the HokUS-10 scoring system.

\section{Conclusion}

24 The inter- and intra-reliabilities of HokUS-10 might be acceptable for the assessment of 25 SOS/VOD under a suitable unified assessment. 


\section{Acknowledgements}

2 We are grateful to all the patients and health professionals who contributed to the data

3 collection at Hokkaido University Hospital.

4

\section{Authorship}

6 T. I. and M. N. participated in the research design, the writing of the paper, the

7 performance of the research, and data analysis.

8 Y. K. and R. T. participated in the performance of the research.

9 I. Y. and R. T. performed statistical analysis.

10 H. S. participated in the review of the paper.

11 J. S. and S. T. participated in the research design, the data analysis, and the review of the 12 paper.

13 T. T. participated in the research design, data analysis, and the writing and review of the 14 paper.

\section{Compliance with ethical standards}

\section{Conflicts of interest}

9 The authors declare that they have no conflicts of interest.

\section{Ethical statement}

22 This study was approved by the institutional review board (016-0075), and written

23 informed consent was obtained from all study participants in accordance with the World

24 Medical Association Declaration of Helsinki: Ethical principles for medical research

25 involving human subjects, 2013.

\section{Role of the funding source}

28 There is none. 


\section{References}

1) Cheuk DK. Hepatic veno-occlusive disease after hematopoietic stem cell transplantation: prophylaxis and treatment controversies. World J Transplant. 2012;2:27-34.

2) Coppell JA, Richardson PG, Soiffer R, et al. Hepatic veno-occlusive disease following stem cell transplantation: incidence, clinical course, and outcome. Biol Blood Marrow Transplant. 2010;16:157-68.

3) Lassau N, Leclere J, Auperin A, et al. Hepatic veno-occlusive disease after myeloablative treatment and bone marrow transplantation: value of gray-scale and Doppler US in 100 patients. Radiology. 1997;204:545-52.

4) Lassau N, Auperin A, Leclere J, et al. Prognostic value of doppler-ultrasonography in hepatic veno-occlusive disease. Transplantation. 2002;74:60-6.

5) Brown BP, Abu-Yousef M, Farner R, et al. Doppler sonography: a noninvasive method for evaluation of hepatic venocclusive disease. AJR Am J Roentgenol. 1990;154:721-4.

6) Herbetko J, Grigg AP, Buckley AR, et al. Venoocclusive liver disease after bone marrow transplantation: findings at duplex sonography. AJR Am J Roentgenol. 1992;158:1001-5.

7) Teefey SA, Brink JA, Borson RA, et al. Diagnosis of venoocclusive disease of the liver after bone marrow transplantation: value of duplex sonography. AJR Am J Roentgenol. 1995;164:1397-401.

8) Schiavetti A, Matrunola M, Varrasso G, et al. Ultrasound in the management of hepatic veno-occlusive disease in three children treated with dactinomycin and vincristine. Pediatr Hematol Oncol. 1996;13:521-9.

9) Kriegshauser JS, Charboneau JW, Letendre L. Hepatic venocclusive disease after bone-marrow transplantation: diagnosis with duplex sonography. AJR Am J Roentgenol. 1988;150:289-90.

10) McCarville MB, Hoffer FA, Howard SC, et al. Hepatic veno-occlusive disease in children undergoing bone-marrow transplantation: usefulness of sonographic findings. Pediatr Radiol. 2001;31:102-5.

11) Park JE, Choi YH, Cheon JE, et al. Gallbladder wall oedema and ascites are independent predictors of progression to hepatic veno-occlusive disease for children with hematopoietic stem cell transplantation. Eur Radiol. 2018;28:2291-8.

12) Mohty M, Malard F, Abecassis M, et al. Revised diagnosis and severity criteria for sinusoidal obstruction syndrome/veno-occlusive disease in adult patients: a 
new classification from the European Society for Blood and Marrow Transplantation. Bone Marrow Transplant. 2016;51:906-12.

13) Kammersgaard MB, Kielsen K, Heilmann C, et al. Assessment of the proposed EBMT pediatric criteria for diagnosis and severity grading of sinusoidal obstruction syndrome. Bone Marrow Transplant. 2019;54:1406-18.

14) Nishida M, Kahata K, Hayase E, et al. Novel ultrasonographic scoring system of sinusoidal obstruction syndrome after hematopoietic stem cell transplantation. Biol Blood Marrow Transplant. 2018;24:1896-900.

15) Corbacioglu S, Kernan NA, Pagliuca A, et al. Incidence of anicteric veno-occlusive disease/sinusoidal obstruction syndrome and outcomes with defibrotide following hematopoietic cell transplantation in adult and pediatric patients. Biol Blood Marrow Transplant. 2020;26:1342-9.

16) Mahadeo KM, Bajwa R, Abdel-Azim H, et al. Diagnosis, grading, and treatment recommendations for children, adolescents, and young adults with sinusoidal obstructive syndrome: an international expert position statement. Lancet Haematol. 2020;7:e61-72.

17) Trenker C, Burchert A, Schumacher C, et al. Pathologic hepatic contrast-enhanced ultrasound pattern in patients undergoing allogeneic Stem cell transplantation. Ultrasound Med Biol. 2020;46:1865-71.

18) Cairo MS, Cooke KR, Lazarus HM, et al. Modified diagnostic criteria, grading classification and newly elucidated pathophysiology of hepatic SOS/VOD after haematopoietic cell transplantation. Br J Haematol. 2020.

19) Bonifazi F, Barbato F, Ravaioli F, et al. Diagnosis and treatment of VOD/SOS after allogeneic hematopoietic stem cell transplantation. Front Immunol. 2020;11:489.

20) Moriyasu F, Ban N, Nishida O, et al. Clinical application of an ultrasonic duplex system in the quantitative measurement of portal blood flow. J Clin Ultrasound. 1986;14:579-88.

21) Landis JR, Koch GG. The measurement of observer agreement for categorical data. Biometrics. 1977;33:159-74.

22) Ignee $A$, Boerner N, Bruening $A$, et al. Duplex sonography of the mesenteric vessels--a critical evaluation of inter-observer variability. Z Gastroenterol. 2016;54:304-11.

23) Dietrich CF, Trenker C, Fontanilla T, et al. New ultrasound techniques challenge the diagnosis of sinusoidal obstruction syndrome. Ultrasound Med Biol. 2018;44:2171-82. 
$124)$ Terminology and Diagnostic Criteria Committee, Japan Society of Ultrasonics in 2 Medicine. Standard method for ultrasound evaluation of carotid artery lesions. J Med Ultrason (2001). 2009;36:219-26. 


\section{Figure captions}

Fig. 1 Gallbladder thickening. B-mode ultrasonography shows stratified thickening $(\geq 6$ $\mathrm{mm})$.

Fig. 2 Moderate-to-severe ascites in the Douglas pouch (a), subhepatic space (b), and spleno-renal interspace (c).

*Catheter and balloon in the urinary bladder, $\dagger$ Liver, $\ddagger$ Spleen

Fig. 3 Dilatation of the paraumbilical vein ( $\geq 2 \mathrm{~mm}$ ) and the appearance of hepatofugal flow can be seen. 
Table 1. HokUS-10

\begin{tabular}{ccc}
\hline Parameters & Description & Points \\
\hline Hepatic left lobe vertical diameter & $\geq 70 \mathrm{~mm}$ & 1 \\
Hepatic right lobe vertical diameter & $\geq 110 \mathrm{~mm}$ & 1 \\
Gallbladder wall thickening & $\geq 6 \mathrm{~mm}$ & 1 \\
PV diameter & $\geq 12 \mathrm{~mm}$ & 1 \\
PUV diameter & $\geq 2 \mathrm{~mm}$ & 2 \\
Amount of ascites & Mild & 1 \\
& Moderate to severe & 2 \\
PV mean velocity & $<10 \mathrm{~cm} / \mathrm{s}$ & 1 \\
Direction of PV flow & Congestion or hepatofugal flow & 1 \\
Appearance of PUV blood flow signal & Yes (Congestion or hepatofugal flow) & 2 \\
Proper hepatic artery RI & $\geq 0.75$ & 1 \\
\hline
\end{tabular}

RI was calculated by the formula of Vmax - Vmin / Vmax.

PV, portal vein; PUV, paraumbilical vein; PV mean velocity, time-averaged flow velocity (TAV); RI, resistive index 
Table 2. Characteristics of the study population

\begin{tabular}{|c|c|c|c|}
\hline & $\begin{array}{l}\text { Healthy volunteers } \\
\qquad(n=24)\end{array}$ & $\begin{array}{l}\text { Patients } \\
(n=40)\end{array}$ & $\begin{array}{l}\text { Total } \\
(n=64)\end{array}$ \\
\hline Gender (male/female) & $16 / 8$ & $21 / 19$ & $37 / 27$ \\
\hline Age, years & $39(24-65)$ & $58(21-85)$ & $52(21-85)$ \\
\hline Weight, kg & $66(40-89)$ & $60(37-114)$ & $64(37-114)$ \\
\hline Height, cm & $167(153-181)$ & $163(136$ - 183) & $165(136$ - 183) \\
\hline BMI, kg/m² & $22.8(16.0$ - 28.2) & $23.2(15.8-40.0)$ & $22.8(15.8-40.0)$ \\
\hline $\begin{array}{l}2^{\text {nd }} \text { time US (day from } \\
1^{\text {st }} \text { study) }\end{array}$ & $2(1-6)$ & - & - \\
\hline \multicolumn{4}{|l|}{ Clinical backgrounds } \\
\hline HBV positivity & - & $16(40.0 \%)$ & - \\
\hline HCV positivity & - & $7(17.5 \%)$ & - \\
\hline HEV positivity & - & $1(2.5 \%)$ & - \\
\hline NAFLD & - & $4(10.0 \%)$ & - \\
\hline ALD & - & $2(5.0 \%)$ & - \\
\hline $\mathrm{AIH}$ & - & $2(5.0 \%)$ & - \\
\hline Others & - & 8 (20.0\%) & - \\
\hline
\end{tabular}

All values are expressed as median (range), unless otherwise noted as $\mathrm{n}(\%)$ for number (frequency) BMI, body mass index; HBV, hepatitis B virus; HCV, hepatitis C virus; HEV, hepatitis E virus; NAFLD, non-alcoholic fatty liver disease; ALD, alcoholic liver disease; AIH, autoimmune hepatitis; Others included primary biliary cholangitis, Idiopathic portal hypertension, drug induced liver disease, and thrombocytopenia, anasarca, fever, reticulin fibrosis and organomegaly syndrome, and idiopathic cirrhosis 
Table 3. Inter-operator reliability of HokUS-10 in healthy volunteers $(n=24)$

\begin{tabular}{|c|c|c|c|c|c|}
\hline \multirow{2}{*}{ Parameters } & \multirow[b]{2}{*}{$\begin{array}{c}\text { Concordance } \\
\text { rate }\end{array}$} & \multirow[b]{2}{*}{ Agreement } & \multicolumn{3}{|c|}{ Sonographers (US experiences) } \\
\hline & & & A (33-years) & B (7-years) & C (2-years) \\
\hline Hepatic left lobe vertical diameter (mm) & $96 \%$ & 0.92 & $48.6(25.9-82.0)$ & $49.1(30.4-80.1)$ & $53.0(31.2-83.0)$ \\
\hline Hepatic right lobe vertical diameter (mm) & $71 \%$ & 0.57 & $104.6(85.9-133.4)$ & $101.3(87.3-122.9)$ & $104.9(84.0$ - 132.4) \\
\hline Gallbladder wall thickening (mm) & $100 \%$ & NA* & $1.2(0.8-2.9)$ & $1.3(0.8-2.7)$ & $1.4(0.8-2.5)$ \\
\hline PV diameter (mm) & $88 \%$ & NA & $9.7(6.0-12.3)$ & $10.2(7.8-12.4)$ & $9.4(6.3-11.7)$ \\
\hline PUV diameter (mm) & $100 \%$ & NA* & $0.8(0.4-1.2)$ & $0.9(0.7-1.1)$ & $0.9(0.5-1.5)$ \\
\hline Amount of ascites (points) & $83 \%$ & 0.63 & $0(0-1)$ & $0(0-1)$ & $0(0-1)$ \\
\hline PV mean velocity (cm/s) & $63 \%$ & NA & $14.2(9.7-23.2)$ & $11.6(7.7-34.2)$ & $14.3(8.4-42.0)$ \\
\hline Direction of PV flow (points) & $100 \%$ & NA* & $0(0-0)$ & $0(0-0)$ & $0(0-0)$ \\
\hline Appearance of PUV blood flow signal (points) & $100 \%$ & NA* & $0(0-0)$ & $0(0-0)$ & $0(0-0)$ \\
\hline Proper hepatic artery RI & $58 \%$ & 0.16 & $0.69(0.50-0.85)$ & $0.73(0.63-0.85)$ & $0.69(0.46-0.81)$ \\
\hline HokUS-10 [cut off value 5] & $100 \%$ & 0.63 & $1(0-4)$ & $1(0-4)$ & $1(0-3)$ \\
\hline Examination time (minutes) & - & - & $9.8(5.5-15.9)$ & $8.2(5.8-15.4)$ & $12.6(5.9-23.2)$ \\
\hline
\end{tabular}

Values are expressed as median (range). Agreement, each item of HokUS-10 was evaluated by the Fleiss' Kappa coefficient, and whether the cutoff value was 5 or more was evaluated with the Krippendorff's alpha coefficient. RI was calculated by the formula of $V_{\max }-V_{\min } / V_{\max }$.

NA, not available (NA*, Kappa coefficient was inestimable because the degree of match was too high); PV, portal vein; PUV, paraumbilical vein; PV mean velocity, time-averaged flow velocity (TAV); RI, resistive index. 
Table 4. Intra-operator reliability of HokUS-10 in healthy volunteers $(n=24)$

\begin{tabular}{|c|c|c|c|c|}
\hline \multirow{2}{*}{ Parameters } & \multirow[b]{2}{*}{$\begin{array}{l}\text { Concordance } \\
\text { rate }\end{array}$} & \multirow[b]{2}{*}{ Kappa } & \multicolumn{2}{|c|}{ Sonographer B (7-years) } \\
\hline & & & First scan & Second scan \\
\hline Hepatic left lobe vertical diameter (mm) & $96 \%$ & 0.86 & $49.1(30.4-80.1)$ & $48.2(28.6-78.1)$ \\
\hline Hepatic right lobe vertical diameter (mm) & $100 \%$ & 1.00 & $101.3(87.3-122.9)$ & $98.7(88.6-126.2)$ \\
\hline Gallbladder wall thickening (mm) & $100 \%$ & NA* & $1.3(0.8-2.7)$ & $1.3(0.8-2.7)$ \\
\hline PV diameter (mm) & $92 \%$ & 0.46 & $10.2(7.8-12.4)$ & $10.0(8.4-12.1)$ \\
\hline PUV diameter (mm) & $100 \%$ & NA* & $0.9(0.7-1.1)$ & $0.8(0.5-1.4)$ \\
\hline Amount of ascites (points) & $96 \%$ & 0.88 & $0(0-1)$ & $0(0-1)$ \\
\hline PV mean velocity $(\mathrm{cm} / \mathrm{s})$ & $88 \%$ & 0.73 & $11.6(7.7-34.2)$ & $11.7(7.1-22.0)$ \\
\hline Direction of PV flow (points) & $100 \%$ & NA* & $0(0-0)$ & $0(0-0)$ \\
\hline Appearance of PUV blood flow signal (points) & $100 \%$ & NA* & $0(0-0)$ & $0(0-0)$ \\
\hline Proper hepatic artery RI & $88 \%$ & 0.75 & $0.73(0.63-0.85)$ & $0.72(0.64-0.83)$ \\
\hline HokUS-10 [cut off value 5] & $100 \%$ & 0.87 & $1(0-4)$ & $1(0-4)$ \\
\hline Examination time (minutes) & - & - & $8.2(5.8-15.4)$ & $9.4(5.3-14.0)$ \\
\hline
\end{tabular}

Values are expressed as median (range). The RI was calculated by the formula of $\mathrm{V}_{\max }-\mathrm{V}_{\min } / \mathrm{V}_{\max }$.

Kappa, weighted Kappa coefficient; NA, not available (NA*, Kappa coefficient was inestimable because the degree of match was too high); PV, portal vein; PUV, paraumbilical vein; PV mean velocity, time-averaged flow velocity (TAV); RI, resistive index. 
Table 5. Inter-operator reliability of three items in the entire study population $(n=64)$

\begin{tabular}{ccccc}
\hline & & & \multicolumn{2}{c}{ Sonographers (US experiences) } \\
\cline { 4 - 5 } Parameters & $\begin{array}{c}\text { Concordance } \\
\text { rate }\end{array}$ & Kappa & A (33-years) & B (7-years) \\
\hline Gallbladder wall thickening (mm) & $98 \%$ & 0.79 & $1.1(0.6-10.1)$ & $1.3(0.8-12.8)$ \\
Amount of ascites (points) & $91 \%$ & 0.85 & $0(0-2)$ & $0(0-2)$ \\
Appearance of PUV blood flow signal (points) & $98 \%$ & 0.92 & $0(0-2)$ & $0(0-2)$ \\
\hline
\end{tabular}

Values are expressed as median (range)

Kappa, weighted Kappa coefficient; PUV, paraumbilical vein 
Table 6. Re-assessments in healthy volunteers $(n=24)$

\begin{tabular}{|c|c|c|c|c|c|c|}
\hline \multirow[b]{3}{*}{ Parameters } & \multicolumn{2}{|c|}{ Images captured by Sonographer A } & \multicolumn{2}{|c|}{ Images captured by Sonographer B } & \multicolumn{2}{|c|}{ Images captured by Sonographer C } \\
\hline & \multicolumn{2}{|c|}{ measurement } & \multicolumn{2}{|c|}{ measurement } & \multicolumn{2}{|c|}{ measurement } \\
\hline & Sonographer A & Sonographer D & Sonographer B & Sonographer D & Sonographer C & Sonographer D \\
\hline Hepatic left lobe vertical diameter (mm) & $48.6(25.9-82.0)$ & $48.5(26.7-82.1)$ & $49.1(30.4-80.1)$ & $50.8(29.4-81.8)$ & $53.0(31.2-83.0)$ & $53.1(30.4-83.4)$ \\
\hline Hepatic right lobe vertical diameter (mm) & $104.6(85.9-133.4)$ & $106.4(83.2-132.6)$ & $101.3(87.3-122.9)$ & $101.3(88.8-126.3)$ & $104.9(84.0-132.4)$ & $106.5(84.8$ - 133.8) \\
\hline Gallbladder wall thickening (mm) & $1.2(0.8-2.9)$ & $1.3(0.8-2.5)$ & $1.3(0.8-2.7)$ & $1.4(0.8-2.4)$ & $1.4(0.8-2.5)$ & $1.4(0.8-2.4)$ \\
\hline PV diameter (mm) & $9.7(6.0-12.3)$ & $10.5(7.1-13.0)$ & $10.2(7.8-12.4)$ & $10.0(8.0-12.3)$ & $9.4(6.3-11.7)$ & $9.7(7.0-12.8)$ \\
\hline PUV diameter (mm) & $0.8(0.4-1.2)$ & $0.7(0.4-1.1)$ & $0.9(0.7-1.1)$ & $0.8(0.5-1.4)$ & $0.9(0.5-1.5)$ & $0.8(0.5-1.2)$ \\
\hline Amount of ascites (points) & $0(0-1)$ & $0(0-1)$ & $0(0-1)$ & $0(0-1)$ & $0(0-1)$ & $0(0-1)$ \\
\hline PV mean velocity $(\mathrm{cm} / \mathrm{s})$ & $14.2(9.7-23.2)$ & $15.8(10.9-29.2)$ & $11.6(7.7-34.2)$ & $12.9(7.9-42.1)$ & $14.3(8.4-42.0)$ & $15.0(10.5-52.2)$ \\
\hline Direction of PV flow (points) & $0(0-0)$ & $0(0-0)$ & $0(0-0)$ & $0(0-0)$ & $0(0-0)$ & $0(0-0)$ \\
\hline Appearance of PUV blood flow signal & $0(0-0)$ & $0(0-0)$ & $0(0-0)$ & $0(0-0)$ & $0(0-0)$ & $0(0-0)$ \\
\hline Proper hepatic artery RI & $0.69(0.50-0.85)$ & $0.71(0.56-0.87)$ & $0.73(0.63-0.85)$ & $0.72(0.63-0.83)$ & $0.69(0.46-0.81)$ & $0.69(0.50-0.81)$ \\
\hline HokUS-10 [cut off value 5] & $1(0-4)$ & $1(0-4)$ & $1(0-4)$ & $1(0-4)$ & $1(0-3)$ & $1(0-3)$ \\
\hline
\end{tabular}

Values are expressed as median (range). RI was calculated by the formula of $\mathrm{V}_{\max }-\mathrm{V}_{\min } / \mathrm{V}_{\max }$. PV mean velocity by Sonographer A, B, C was time-averaged flow velocity (TAV). The PV mean velocity in Sonographer D was calculated by multiplying the time-averaged maximum velocity (TAMV) by 0.57 . PV, portal vein; PUV, paraumbilical vein; RI, resistive index 
Figue.1

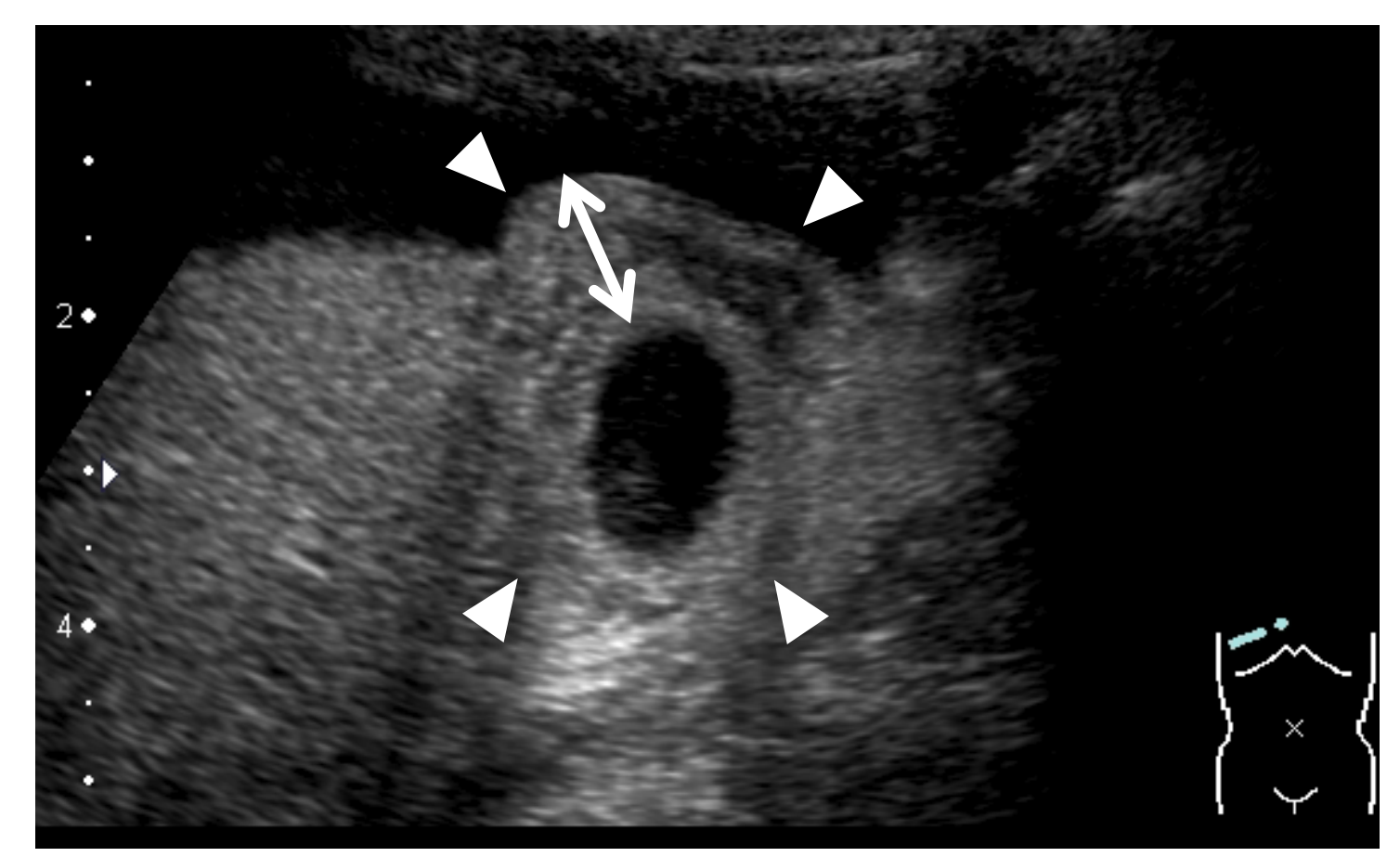


Figue.2

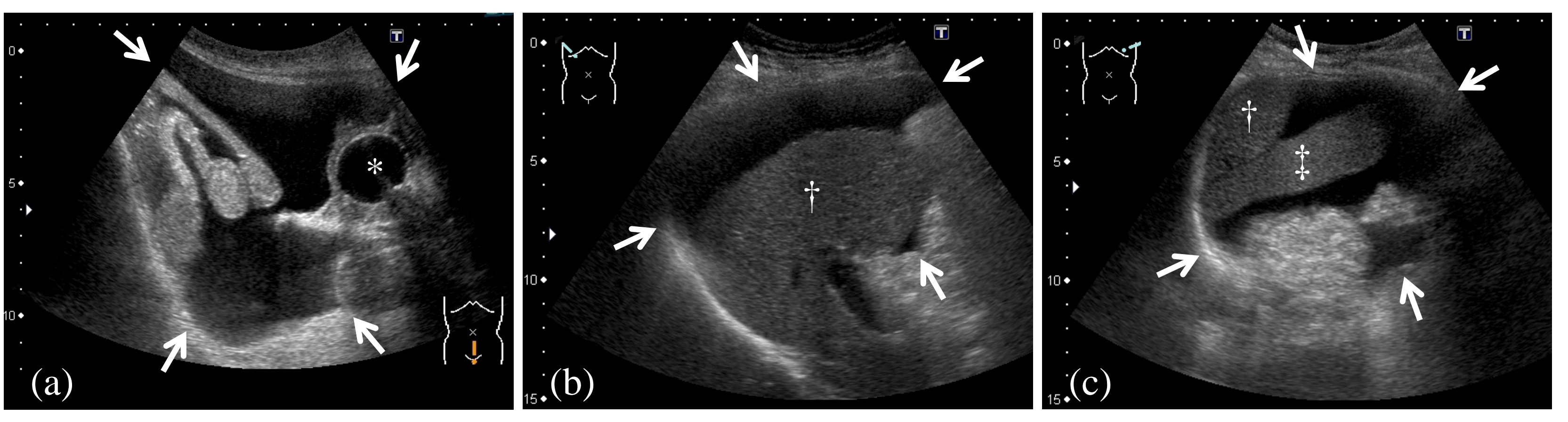


Figue.3

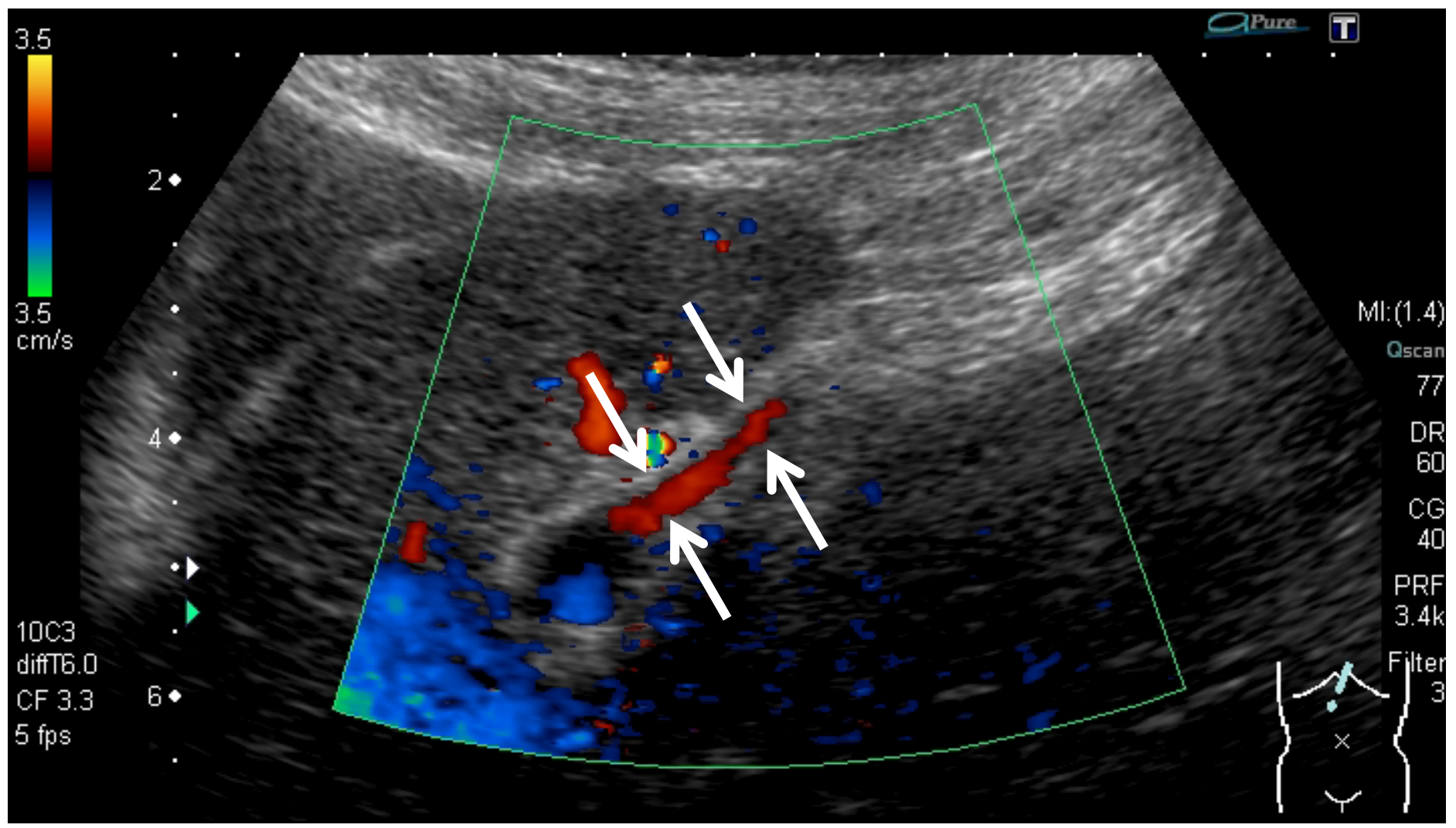

